DOI: 10.17234/SRAZ.65.10

UDK: 811.133.1'367.622:811.163.42

UDK: 811.133.1'243'367.622:371.3

Original scientific paper

Reçu le 29 octobre 2020

Accepté pour la publication le 25 novembre 2020

\title{
Sur le genre et le nombre des noms en français et en croate
}

\author{
Lidija Orešković Dvorski \\ Sanja Šoštarić \\ Faculté de philosophie et lettres, Université de Zagreb \\ lodvorsk@ffzg.hr \\ ssostari@ffzg.hr
}

Parmi de nombreux aspects du nom qui demeurent problématiques dans la langue française, notre article se focalisera sur les sujets suivants : genre et sexe, singularia et pluralia tantum, noms distingués par le genre, changement de sens résultant $d u$ changement de genre ou de nombre, nombre et dénombrabilité. L'apprentissage d'une langue étrangère étant influencé par la langue maternelle, nous analyserons aussi les spécificités du nom dans la langue croate.

Mots-clés : nom, genre, nombre, français, croate

\section{Introduction}

En définissant le nom, Le Bon usage souligne qu'il est porteur d'un genre et susceptible de varier en nombre, parfois en genre. Il définit le genre comme « une propriété du nom, qui le communique, par le phénomène de l'accord, au déterminant, à l'adjectif épithète ou attribut, parfois au participe passé, ainsi qu'au pronom représentant le nom ». (Grevisse 2016 : 637) Ensuite, il indique qu'il y a deux genres : le masculin et le féminin.

En ce qui concerne le croate, parmi les caractéristiques grammaticales du nom, on distingue le genre (masculin, féminin, neutre), le nombre (singulier, pluriel) et le cas (nominatif, génitif, datif, accusatif, vocatif, instrumental, locatif). Par ailleurs, les noms sont regroupés en trois classes de déclinaisons, déterminées en fonction de leur désinence au génitif singulier, à savoir les classes $a$, e et $i$. (Barić 2005 : 103). 


\section{Genre}

\section{II.1. Noms inanimés}

L'étude du genre révèle plusieurs facettes intéressantes à analyser : rapport genre - sexe, noms homonymes distingués par le genre, règles pour reconnaître le genre et noms douteux. Ces aspects du genre peuvent être envisagés dans deux optiques : théorique et pratique, à savoir du point de vue des linguistes et grammairiens ou du point de vue des locuteurs natifs « ordinaires » et des apprenants de français langue étrangère.

En ce qui concerne les linguistes, leur débat sur la relation entre genre grammatical et différence sexuelle date du commencement du $\mathrm{XX}^{\mathrm{e}}$ siècle. Selon leurs travaux, le genre grammatical est dit motivé s'il correspond au sexe, pris dans un sens naturel pour les animés et métaphorique pour les inanimés. Si ce n'est pas le cas, il est désigné comme arbitraire.

D'autre part, les locuteurs dits ordinaires ne s'intéressent pas au sujet de l'aspect théorique des genres. À première vue, le locuteur natif du français ne met pas en cause la répartition des noms en deux groupes : il trouve cette opposition binaire plutôt « naturelle », d'autant plus qu'elle correspond - au moins pour les animés - à la différence sexuelle. C'est en apprenant d'autres langues qu'il se rend compte que certaines langues possèdent trois genres (p. ex. le russe) ou ne comportent pas la catégorie du genre au sens qui lui est donné dans sa langue maternelle (p. ex. l'anglais).

Dans les lignes qui suivent nous essaierons d'expliquer comment les francophones natifs distinguent le genre grammatical en français, en nous focalisant sur les noms inanimés. Le Bon usage indique que leur genre est arbitraire, à savoir qu'il n'est pas déterminé par leur sens. Cette constatation pourrait être illustrée par quelques exemples appartenant au même champ sémantique (p. ex. le ruisseau, la rivière, le fleuve) ou par la comparaison entre le français et le croate (fr. le soleil, m. ; la lune, f. - cr. sunce, n. ; mjesec, m.). En outre, beaucoup de noms ont changé de genre au cours de l'histoire : tandis qu'autrefois affaire, dent, erreur étaient des noms masculins et doute, poison, silence féminins, aujourd'hui c'est l'inverse.

Le locuteur natif ne mémorise pas le genre de chaque mot séparément ; il se sert de différents repères formels ou sémantiques qui l'aident à identifier le genre. Les indices formels regroupent les éléments morphologiques et phonologiques. D'après les expériences linguistiques, le natif associe rapidement certains graphèmes (-is, -ment, -isme, etc.) au masculin et d'autres (-ie, -ée, -ette, etc.) au féminin (Jeanmaire 2010 : 72-73). Les indices sémantiques portent sur le sens du nom. Le natif intègre très tôt quelques indications utiles à reconnaître le genre : par exemple que sont féminins les noms de sciences, de disciplines ou d'arts et que sont masculins les noms de couleurs, de langues, d'arbres, etc. 
Tandis que le natif reconnaît spontanément les terminaisons qui peuvent indiquer le genre du nom parce qu'il s'appuie sur sa compréhension de la signification des noms ainsi que sur la comparaison avec d'autres noms ayant la même forme, pour les apprenants d'une langue étrangère le genre représente un élément extrêmement difficile à maîtriser. Par conséquent, le locuteur non-natif doit se servir de différentes stratégies d'apprentissage du genre : il apprend les règles régissant le genre des noms et mémorise parfois le genre nom par nom. L'apprentissage d'une langue étrangère est souvent influencé par la langue maternelle. Ainsi les croatophones attribuent-ils incorrectement le genre féminin aux noms livre, groupe et domaine parce que ceux-ci sont du genre féminin en croate (knjiga, grupa, domena).

Les remarques mentionnées plus haut pourraient conduire à conclure que le natif distingue sans faute le genre de chaque nom. Toutefois, en l'absence d'indications contextuelles, il peut hésiter sur le genre de certains mots. En français, les hésitations sont fréquentes quand le nom commence par une voyelle ou un $h$ muet (abîme, m., idylle, f., héliotrope, m., etc.) qui neutralisent les déterminants (1', son). D'autre part, certains homonymes changent de sens selon qu'ils sont masculins ou féminins : le / la livre, mémoire, mode, tour, vase, etc.

Le croatophone natif peut, lui aussi, se trouver confronté à certains problèmes dans l'identification des genres. Par exemple, les noms bol, čar, glad et quelques noms en -ež (gnjilež, svrbež, trulež) suscitent des hésitations entre masculin et féminin et peuvent se décliner selon deux déclinaisons : en - $i$ et en $-a$. Pour certains d'entre eux, le changement de genre s'accompagne d'un changement de signification : par exemple bol au masculin désigne la douleur physique tandis qu'au féminin il désigne l'aspect psychique de la douleur. Il y a aussi des noms qui hésitent entre le masculin et le neutre (p. ex. finale, jugo, kino) sans changer de sens.

\section{II.2. Noms animés}

Dans cette catégorie de noms le genre n'est pas arbitraire puisqu'il est déterminé par le sexe des êtres humains désignés. En ce qui concerne les noms d'animaux, on distingue ceux dont le genre est en rapport avec le sexe de l'animal (p. ex. fr. le canard - la cane, cr. patak, m. - patka, f.) et ceux qui possèdent un genre unique indépendamment de la distinction sexuelle (p. ex. fr. la baleine, le puma ; cr. kit, m., puma, f.). En examinant son fichier personnel contenant 7889 noms d'animaux, Jean Dubois a trouvé 6143 masculins et 1700 féminins. Cependant, il indique qu'il n'y a que 23 espèces animales desservies par deux noms de genres différents selon le sexe. Dubois constate qu'il s'agit d'un nombre extrêmement faible, de noms « appartenant fondamentalement à la catégorie des mammifères (mais pas seulement) et fondamentalement aussi aux catégories de l'élevage et de la chasse » Dubois (1989: 88). Il faut y ajouter une vingtaine de termes d'origine différente (p. ex. le coq - la poule, le taureau - la vache, le lièvre - la hase) qui s'articulent, eux aussi, autour de l'opposition mâle - femelle. 
Dans les chapitres portant sur le féminin des noms animés, les grammairiens français indiquent souvent qu'il se marque : a. par addition d'un -e à la forme masculine (ami, amie), b. par modification ou addition de suffixe (menteur, menteuse) ou c. par un nom de même radical que celui du masculin (neveu, nièce) ou un nom dont le radical est complètement différent de celui du masculin (oncle, tante).

En ce qui concerne les grammairiens du croate, la distinction animé - inanimé ne figure pas dans leur classification des noms car elle n'est marquée qu'à l'accusatif singulier masculin, mais ils en parlent en expliquant la catégorie du genre. Ainsi Eugenija Barić (2005 : 101) indique que le genre est dans une certaine mesure lié à la signification du nom : les noms désignant un être masculin sont du genre masculin et ceux désignant un être féminin du genre féminin. Il n'y a que quelques exceptions à cette règle (p. ex. momče, djevojče, curičak, etc.). Ensuite, Barić ajoute que le genre des noms désignant des choses ou des êtres dont on ne connaît pas le sexe, ou dont le sexe n'est pas important, est déterminé par leur désinence : les noms se terminant par une consonne appartiennent au masculin (pourtant, il y a quelques exceptions : p. ex. riječ, f.), ceux en - $a$ au féminin (sauf les noms désignant un homme : p. ex. tata, m.), tandis que les noms en - o et en - $e$ sont du genre neutre (sauf les hypocoristiques : p. ex. ujo, m.).

Dans les exemples cités le genre du nom est conforme à son sexe. Toutefois, parmi les noms de ce groupe, certains concernent un seul des deux sexes. Par exemple, les noms eunuque, ténor, macho, dandy ne s'appliquent qu'à des hommes, ainsi que les noms désignant certains fonctions, professions et titres (curé, mousquetaire, amiral, etc.). D'autre part, parmi les noms appliqués seulement à des femmes, on trouve nourrice, diva, sage-femme, etc.

En analysant ce problème dans la langue croate, Tatjana Pišković (2011 : 166) emploie le terme " noms défectifs » (ils ne désignent qu'un sexe) parmi lesquels elle distingue feminina tantum (babica, dojilja, trudnica, etc.) et masculina tantum (eunuh, as, mušketir, etc.).

\section{Nombre}

Le nombre - propriété inhérente du nom (Marković 2012 : 233), mais aussi, du fait de l'accord, propriété grammaticale de tous les éléments variables à l'intérieur du groupe nominal ainsi que du verbe s'accordant avec le sujet - représente l'un des mécanismes cognitifs fondamentaux dans la catégorisation de la réalité. Plus précisément, le nombre grammatical traduit la capacité et le besoin de déterminer la quantité des référents extralinguistiques. A propos de la mise au pluriel, Charaudeau (1992 : 240) distingue deux fonctions : une fonction de quantification, à travers la forme plurielle de certains mots, et une fonction d'accord, censée marquer morphologiquement la cohérence sémantique de la pluralité dans l'énoncé. 
La plupart des langues expriment la quantité par des moyens grammaticaux, lexicaux ou bien syntaxiques. Les langues croate et française ont grammaticalisé les quantités un comme singulier et plus d'un comme pluriel. Cette quantification indéfinie, imprécise, dite grammaticale, exprimée par le nombre en tant que catégorie morphologique, s'oppose à la quantification définie, précise, appelée numérique, exprimée par des adjectifs numéraux selon Pranjković (2013 : 35-36), qui met en évidence au moins deux autres types de quantification : lexico-grammaticale, concernant les noms collectifs, et lexicale, liée aux adjectifs indéfinis, adverbes et locutions quantitatives (fr. plusieurs, un peu de, beaucoup de, etc. ; cr. nekoliko, malo, puno, etc.). Le texte qui suit porte sur les différences de la quantification grammaticale en français et croate et fait ressortir les difficultés qui en relèvent.

\section{III.1. Formation du pluriel des noms}

En général, la forme non marquée du nom, telle qu'on la trouve dans les dictionnaires, est le singulier.

En français écrit, le pluriel d'un substantif se marque par l'adjonction d'un -s (ami - amis) ou d'un - $x$ (cadeau - cadeaux) à la forme du singulier (Grevisse 2016 : 739). Du point de vue phonétique (ibid. : 737), le plus grand nombre des noms au pluriel sont prononcés comme au singulier (chat-chats), le pluriel étant assuré par les déterminants (mes camarades) et grâce aux liaisons (mes bons amis).

En croate, écrit et parlé, pour former le pluriel, on dispose des morphèmes polyfonctionnels : les trois catégories du nom (genre, nombre et cas) sont exprimées par une seule désinence (Barić et al. 2005 : 102), par exemple - $i$ dans zakon-i, pour le nominatif et le vocatif du pluriel ou kost-i, pour le nominatif, le génitif et l'accusatif du pluriel, radost-i, pour le nominatif et l'accusatif du pluriel, ensuite le morphème $-e$ dans sob-e, en tant que nominatif, accusatif et vocatif du pluriel, ou le morphème - $a$ dans mor- $a$ formant le nominatif, l'accusatif et le vocatif du pluriel. Un grand nombre de noms est caractérisé dans toutes les formes du pluriel par les infixes -ov ou -ev (ibid : 104), insérés à l'intérieur de la racine (zid-ov-i; kralj-ev-i).

A part les noms qui se prêtent aux moyens grammaticaux susmentionnés dans la formation du pluriel, il faut également mentionner d'une part ceux qui exigent l'utilisation de formes supplétives pour exprimer l'opposition en nombre (fr. fleur-bouquet, abeille-essaim, chanteur-chœur ; cr. čovjek-ljudi, pjevač-zbor, pčela-roj, etc.), et d'autre part ceux qui ne varient pas en nombre (fr. gens; cr. leđa).

\section{III.2. Nombre grammatical et dénombrabilité}

La propriété grammaticale du nombre est liée d'une façon incontournable à un autre phénomène, connu sous le terme de dénombrabilité. Il s'agit d'une catégorie sémantique universelle (Znika 2002 : 56) qui nous permet de distinguer les noms comptables, ou dénombrables, et les noms non comptables ou massifs. Tandis que la plupart des substantifs sont comptables et susceptibles d'être employés au singulier de même qu'au pluriel (cheval - chevaux), un assez grand nombre de noms, qualifiés de massifs, ne correspond pas à cette dualité de forme 
(sérénité - *sérénités). Après avoir été introduite par Jespersen (1924 : 190-205), cette distinction a suscité beaucoup d'attention parmi les linguistes, ce qui a abouti à une profusion de travaux adoptant différents points de vue et soulevant de nombreuses questions concernant, par exemple, le statut, l'emploi, la terminologie ou les (sous-)classifications de ces noms morphologiquement défectifs.

Bien qu'indissociable de la catégorie du nombre grammatical, la dénombrabilité en tant qu'un des aspects sémantiques fondamentaux du nom n'est pas suffisamment abordée dans les grammaires françaises et croates consultées (Barić et al ; Charaudeau, Grevisse), ce qui, à en croire une recherche menée par Tafra en 2007, suscite une incompréhension de ces catégories de la part des apprenants, des étudiants et même des enseignants croatophones.

\section{III.3. Déficit(s) de dénombrabilité}

A la différence des noms dits prototypiques avec paradigme complet (ayant les formes du singulier et du pluriel), il existe un ensemble hétérogène de noms dits non comptables caractérisés par l'absence de l'une des deux formes ou par la modification de sens plus ou moins considérable apportée par le changement du nombre. Dans les trois sections suivantes, nous nous efforçons d'esquisser leurs types les plus importants reflétant en même temps les difficultés de délimitation et de chevauchement des catégories du nombre et de la dénombrabilité ainsi que celles qui reposent sur la polysémie grammaticale de la catégorie du nombre.

\section{III.3.1. Noms sans singulier}

Certains noms, connus sous le terme de pluralia tantum ou pluriels lexicaux, ne s'emploient qu'au pluriel : les uns expriment une pluralité d'êtres ou d'objets, tandis que d'autres concernent des ensembles vagues dans lesquels il est difficile d'identifier des unités. Voilà quelques exemples symétriques de noms sans singulier en français et en croate : pierreries - drago kamenje, finances - financije, oreillons -zaušnjaci, etc. Par ailleurs, il est possible de trouver des noms illustrant une asymétrie quant à la variation en nombre entre les langues française et croate, tel que le nom mœurs, employé toujours au pluriel, dont l'équivalent croate običaji peut s'employer au singulier. Il en va de même pour l'emploi au singulier du nom croate špinat, qui ne correspond pas en nombre à son équivalent français épinards, employé au pluriel. Citons d'autres pluriels lexicaux français et leurs équivalents croates asymétriques au singulier : condoléances - sućut, échecs - šah, alentours - okolica, etc. De même, à certains pluriels lexicaux croates tels que leđa, vrata, grablje, etc. correspondent les noms français au singulier (dos, porte, râteau, etc.).

\section{III.3.2. Noms sans pluriel}

Parmi les noms communs qui s'emploient ordinairement au singulier se distinguent particulièrement deux catégories : les noms massifs qui désignent une substance, une matière, le matériel (argent-srebro, farine-brašno, sucre-šećer) et certains noms collectifs, désignant un ensemble d'êtres ou de choses (clergé-kler, nourriture - hrana, public - publika, mobilier - namještaj). Il faut mentionner aussi 
un grand nombre de noms abstraits qui ne s'emploient pas au pluriel dans leur sens primaire : amour - ljubav, haine - mržnja, patience - strpljenje, fatigue - umor, temps - vrijeme, air - zrak.

\section{III.3.3. D'autres difficultés liées au nombre}

La complexité sous-jacente de cette catégorie fondamentale de toute langue est encore plus accentuée du point de vue croatophone lorsqu'on évoque quelques cas particuliers, comme les noms qui peuvent être employés indifféremment au singulier et au pluriel en français (bien qu'il ne s'agisse pas de synonymes absolus), ce qui n'est pas possible en croate dont les équivalents cités sont des pluriels lexicaux : vêtement(s) - odjeća, escalier(s) -stepenice, orgue(s)-orgulje, etc. Ensuite, les noms se terminant par $-s$ au singulier (concours, puits, propos, remords, cours, corps, etc.) représentent une difficulté due à leur irrégularité formelle au singulier. Le changement de sens provoqué par le changement de nombre des noms français tels que vacance(s), lunette(s), lettre(s) exige une attention particulière ainsi que la conversion de noms massifs en noms comptables (Nicolas 2002 : 113) ou bien la conversion de noms comptables en noms massifs (ibid. 109 ; Kleiber 2014 : 15).

En plus de la problématique issue des déficits de dénombrabilité que nous avons essayé de mettre en évidence dans la présente section, il ne faut pas oublier la polysémie du lexique qui permet à un nom fondamentalement comptable de revêtir le caractère massif, et l'inverse, dans une de ses significations (p. ex. L'amour est le plus beau des sentiments ; J'ai deux amours).

\section{Conclusion}

Bien qu'il n'existe aucune règle absolue pour la détermination du genre des noms français, il y a des régularités formelles (phonético-graphiques et morphologiques) qui indiquent le genre d'un nom désignant un inanimé. En ce qui concerne les animés, on ajoute aux régularités formelles les indices sémantiques liés au sexe du référent. Les locuteurs natifs ont une connaissance implicite et intuitive de ces régularités, ce qui est démontré par plusieurs recherches se proposant de catégoriser des emprunts et des noms fictifs isolés. D'autre part, dans l'apprentissage d'une langue étrangère on peut discerner deux approches opposées : apprendre le genre nom par nom ou apprendre les caractéristiques sémantiques et formelles. En tant qu'enseignantes du français, nous pouvons dire que nos étudiants combinent ces deux approches.

Pour ce qui est du nombre, nous remarquons qu'il s'agit d'une catégorie apparemment simple qui, en réalité, comporte de nombreuses facettes reflétant la relation étroite entre sémantique et grammaire. La polysémie du nombre grammatical et les différences entre langues dans la conception de la réalité extralinguistique mènent à des asymétries entre les noms en français et croate du fait de la grammaticalisation différente du singulier et du pluriel dans ces deux langues. 


\section{Bibliographie}

Barić, Eugenija et al. (2005). Hrvatska gramatika, Zagreb: Školska knjiga.

Charaudeau, Patrick (1992). Grammaire du sens et de l'expression, Paris : Hachette. Dubois, Jean (1989). Le genre dans les noms d'animaux, in : Linx, 21, pp. 87-91. Grevisse, Maurice / Goosse, André (2016). Le Bon Usage, Paris : De Boeck Supérieur. Jeanmaire, Guillaume (2010). Vox populi vox Dei ? L'identification du genre grammatical en français, in: Langue française, 168, pp. 71-86.

Jespersen, Otto (1924). The Philosophy of Grammar, London: George Allen \& Unwin Ltd.

Kleiber, Georges (2014). Massif/comptable. D'une problématique à l'autre, in : Langue française, 183, pp. 3-24.

Marković, Ivan (2012). Uvod u jezičnu morfologiju, Zagreb: Disput.

Nicolas, David (2002). La distinction entre noms massifs et noms comptables : aspects linguistiques et conceptuels, Louvain : Editions Peeters.

Pišković, Tatjana (2011). Gramatika roda, Zagreb: Disput.

Pranjković, Ivan (2013). Gramatička značenja, Zagreb: Matica hrvatska.

Tafra, Branka (2007). Broj i rod ispočetka, in: Filologija, 49, pp. 211-233.

Znika, Marija (2002). Kategorija brojivosti u hrvatskom jeziku, Zagreb: Institut za hrvatski jezik i jezikoslovlje.

\section{O rodu i broju imenica $u$ francuskom i hrvatskom}

Rod i broj, temeljne gramatičke kategorije imenice, dulje su vrijeme predmetom zanimanja velikog broja filozofa i jezikoslovaca. Iako intenzivno proučavani, mnogi aspekti roda i broja još uvijek stvaraju poteškoće, ne samo pri učenju stranog jezika, već i među izvornim govornicima. U radu $O$ rodu $i$ broju imenica $u$ francuskom $i$ hrvatskom daje se pregled nekih otvorenih pitanja kao što su odnos između roda i spola, singularia i pluralia tantum, veza kategorija broja i brojivosti, promjena značenja potaknuta promjenom roda i broja. Posebno se ističu pojedini primjeri nepodudaranja u rodu i/ili broju francuskih i hrvatskih imenica, što kroatofonim studentima često predstavlja izazov pri svladavanju/ učenju francuskog kao stranog jezika.

Ključne riječi: imenica, rod, broj, francuski, hrvatski 